\title{
Metformin Inhibits Esophagus Cancer Proliferation through Upregulation of USP7
}

Yunhua Xu Shun Lu

Department of Shanghai Lung Tumor Clinic Medical Center, Shanghai Chest Hospital, Shanghai Jiao Tong University, Shanghai, China

\section{Key Words}

Metformin • Esophagus cancer • USP7 • mTOR/p70S6K/pS6 signaling • AMPK signaling

\begin{abstract}
Background: Recent population studies suggest that the use of metformin is associated with reduced incidence and improved prognosis of certain cancers. Methods: In the current study, we assessed the effect of metformin on esophagus cancer cells using two cell lines (Eca-109 and TE-1 cells). Results: We found that metformin inhibited growth and decreased expression of cell-cycle regulators in these cells. Treatment with metformin was also associated with activation of AMP kinase and inhibition of $\mathrm{mTOR} / \mathrm{p} 70 \mathrm{~S} 6 \mathrm{~K} / \mathrm{pS} 6$ signaling in both cells. However, inhibition of AMPK signaling has little impact on the anti-proliferative roles of metformin. In addition, we found USP7, a positive regulator of tumor suppressor p53, as a new molecular target of metformin. Esophagus cancer cells can be protected against metformin-induced growth inhibition by small interfering RNA against USP7. Conclusion: These results provide evidence for a mechanism that may contribute to the antineoplastic effects of metformin suggested by recent population studies and justify further work to explore potential roles for it in esophagus cancer prevention and treatment.
\end{abstract}

Copyright $\odot 2013$ S. Karger AG, Basel

\section{Introduction}

Esophagus cancer, which has a particularly high incidence in East Asian countries, commonly has poor prognosis [1]. It is the eighth most common incident cancer and sixth in cancer mortality in the world [2]. Although the current treatment includes radiation therapy, chemotherapy, and esophagogastric resection, many patients with esophageal cancer 
experience progression of disease, indicating that these tumors are resistant to standard therapies [2].

Metformin (1,1-dimethylbiguanide hydrochloride), an oral anti-diabetic drug, is a biguanide usually used in the treatment of type 2 diabetes mellitus with obesity, insulin resistance, and hyperinsulinemia [3-5]. A growing body of evidence from clinical studies and animal models suggests that the primary function of metformin is to decrease hepatic glucose production, mainly by inhibiting gluconeogenesis [6]. Moreover, recent studies carried out using population registries raise the notion that metformin could reduce cancer risk and/ or improve cancer prognosis [7-10]. Besides, an anti-mitogenic effect of metformin has been suggested by in vitro and epidemiological studies $[9,10]$. Metformin was suggested to inhibit cancer cell proliferation and tumor growth in animal models $[11,12]$. For example, in prostate cancer cells, metformin provokes cell-cycle arrest in G0-G1 phase but does not induce apoptosis or autophagy [13]. Moreover, metformin inhibits growth of breast cancer cell lines, blocks cellular transformation in an inducible model system, and has anti-tumor effects in mouse xenografts $[11,14]$. However, the functions of metformin on the esophagus cancer cells remain unexplored.

At the molecular level, metformin regulates the AMP-activated protein kinase signaling, consequently inhibits the mTOR pathway to suppress energy-consuming pathways and protein synthesis $[15,16]$. In agreement, AMPK inhibition abolishes the anti-proliferative effects of metformin in ovarian and breast cancer cells $[17,18]$. However, it was recently reported that metformin can act independent of AMPK signaling. Metformin could inhibit hepatic glucose production in both wild-type and AMPK $\alpha 1 \alpha 2$-deficient hepatocytes through the inhibition of ATP production [19]. In addition, metformin can act independently of AMPK to downregulate mTOR and, instead, signals through Rag GTPase [20]. Taken together, these results suggest a rather complicated mechanism for the metformin action.

Here, we describe in vitro experiments carried out to investigate the hypothesis that metformin exhibits direct antiproliferative actions on esophagus cancer cells. We also found USP7, a positive regulator of tumor suppressor p53, as a new molecular target of metformin.

\section{Materials and Methods}

\section{Cell cultures}

The esophagus cancer cell line Eca-109 and TE-1 cells were purchased from The Cell Bank of Type Culture Collection of Chinese Academy of Sciences (CAS, Shanghai), and cultured in Dulbecco modified Eagle's medium (DMEM, Gibco, USA) supplemented with 10\% fetal calf serum (Gibco, USA), $100 \mathrm{IU} / \mathrm{ml}$ penicillin and $100 \mathrm{mg} / \mathrm{ml}$ streptomycin(Gibco, USA). Cultures were maintained at $37^{\circ} \mathrm{C}$ in a humidified atmosphere with $5 \% \mathrm{CO}_{2}$.

\section{Cell viability and BrdU incorporation assays}

Cell viability was assessed using the 3-(4,5-dimethylthiazol-2-yl)-2,5-diphenyltetrazolium bromide (MTT) assay (Sigma Chemical Co., St. Louis, MO). After preculture, cells were treated with medium containing different doses of metformin (Sigma) and/or different agents as described in Results and figure legends. MTT assay was performed by incubating the cells with $0.5 \mathrm{mg} / \mathrm{ml}$ MTT for 6 hours at $37 \mathrm{C}$ in $5 \%$ $\mathrm{CO}_{2}$. The formazan product was dissolved in dimethyl sulfoxide, and absorbance was read at $490 \mathrm{~nm}$. A cell proliferationenzyme-linked immunosorbent assay kit (Beyotime, Shanghai) was used to analyze the incorporation of BrdU during DNA synthesis following the manufacturer's protocols. All experiments were repeated at least three times in quadruplicate.

\section{RNA isolation and Real-time PCR}

Total RNAs were isolated from cells by TRIzol reagent (Invitrogen, USA), and reverse transcriptions were performed by Takara RNA PCR kit (Takara, Dalian, China), following the manufacturer's instructions. In order to determine the transcripts of the interest genes, Real-time PCR was performed using a SYBR Green Premix Ex Taq (Takara, Dalian, China) on an ABI 7500 machine. 
Fig. 1. Metformin inhibits cell proliferation in esophagus cancer cells. (A-B) Cell viability was measured by MTT assays in Eca-109 (A) and TE-1 cells (B). Cells were treated with various concentrations of metformin as indicated. (C-D) Cell proliferation activity was measured by BrdU incorporation assays in Eca-109 (C) and TE-1 cells (D).

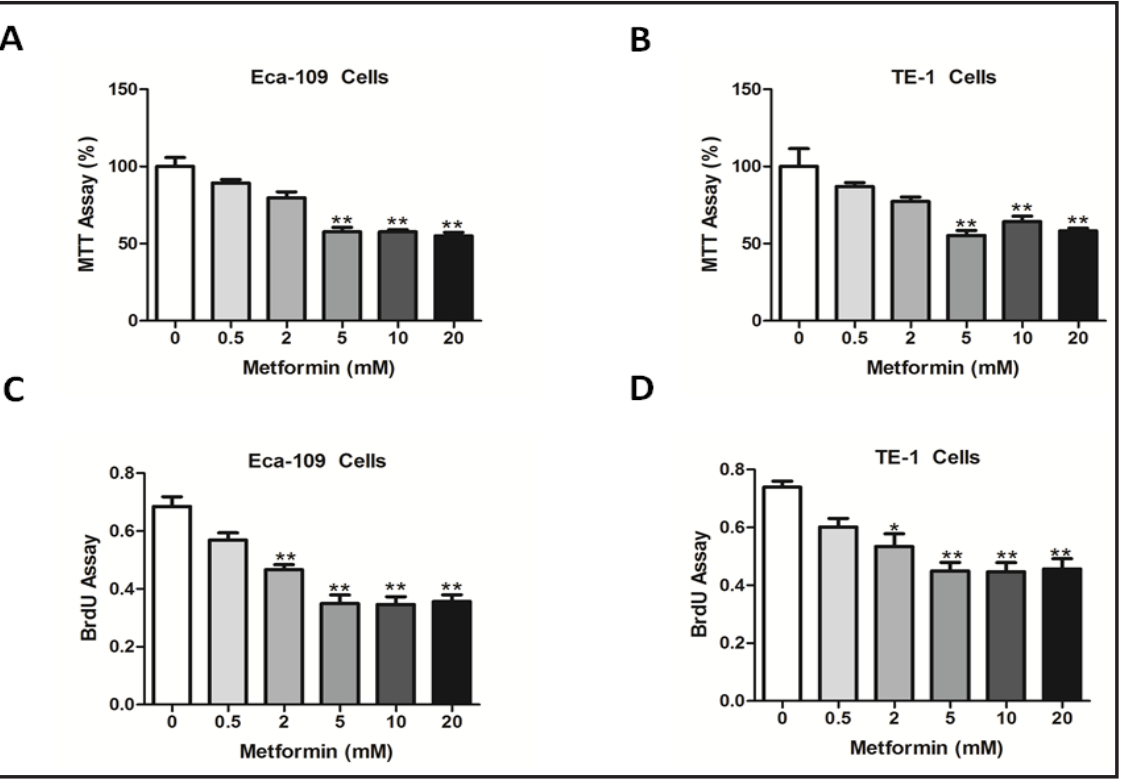

Western blot analysis

Cells after different treatments were lysed with RIPA buffer. An equal amount of protein was subjected to $8 \%$ SDS-PAGE, and separated proteins were transferred to nitrocellulose membranes. The membranes were blocked in $10 \%$ skim milk for 2 hour at room temperature. The immunoblots were incubated overnight at $4{ }^{\circ} \mathrm{C}$ with antibodies. Next day, the membranes were incubated with a horseradish peroxidase-conjugated secondary antibody (Santa Cruz Biotechnology) for 2 hour at room temperature. The immunoreactive bands were detected with chemiluminescence substrate kit (ProteinSimple, Santa Clara, CA) under the Fluor Chem FC2 system. Antibodies were purchased from Abcam (anti- $\beta$ actin, anti-AMPK, anti-ACC, anti-USP7, antiYY1, anti-Mdm2 and anti-p53) or Cell signaling company (anti-p21, anti-p27 and anti-Cyclin D1).

\section{Small interfering RNA (siRNA)}

Cells were transfected with siRNA targeting the AMPK $\alpha 1$ and $\alpha 2$ subunit, the USP7 gene or a negative control (all siRNA oligos from QIAGEN, Valencia, CA) using Lipofectamine 2000 (Invitrogen, USA) as described by the manufacturer's instructions. Cell cultures were incubated for 18 hours with 100nM siRNA before metformin treatment.

\section{Statistical analysis}

Statistical analysis was performed with SPSS version 13.0 software. Numerical data are expressed as mean \pm SEM. Statistical significance is shown as $*(\mathrm{P}<.05)$, ** $(\mathrm{P}<.01)$, or $* * *(\mathrm{P}<.001)$.

\section{Results}

\section{Metformin treatment inhibited cell growth in a dose-dependent manner}

To our knowledge, the effect of metformin on esophagus cancer cells remains unexplored. Thus, we selected two cell lines (Eca-109 and TE-1 cells) to investigate whether metformin has potential anti-proliferation roles. Both cell lines were treated with metformin at several concentrations. After 24 hours of treatment, growth was inhibited in a dose-dependent manner in both cell lines as determined by MTT and BrdU incorporation assays (Fig. 1A1D). Moreover, these results suggested that the concentration of metformin at $5 \mathrm{mM}$ was appropriate in both cell lines. Therefore, $5 \mathrm{mM}$ of metformin was selected for the further analysis of genes expression in both cell lines. 
Fig. 2. Metformin regulates expression of cell-cycle regulators. (A-B) The cell cycle phase of Eca-109 (A) and TE-1 (B) cells treated with vehicle control (Ctrl) or metformin $(5 \mathrm{mM})$ was analyzed by flow cytometry. Cells were labeled for 15 min with PI and immediately analyzed by flow cytometry. Histograms represent the percentage of cells in each phase of the cell cycle (G0/G1, $\mathrm{S}$ and $\mathrm{G} 2 / \mathrm{M}$ ). (C-D) mRNA (C) and protein (D) levels of $\mathrm{p} 21$, p27 and Cyclin D1 were determined by real-time PCR and western blot in Eca-109 cells treated with vehicle control (Ctrl) or metformin (5mM). (EF) mRNA (E) and protein (F) levels of p21, p27 and Cyclin D1 were determined by realtime PCR and western blot in TE-1 cells treated with vehicle control (Ctrl) or metformin (5mM).

Fig. 3. Metformin induces AMPK activation in esophagus cancer cells. (A-B) Western blot analysis of phosphorylated AMPK and ACC in Eca-109 (A) and TE-1 cells (B). Contents of total AMPK, ACC and $\beta$-actin were used as loading controls. (C-D) Western blot analysis of phosphorylated mTOR and S6K in Eca-109 (C) and TE- 1 cells (D). Contents of total mTOR, S6K and $\beta$-actin were used as loading controls.
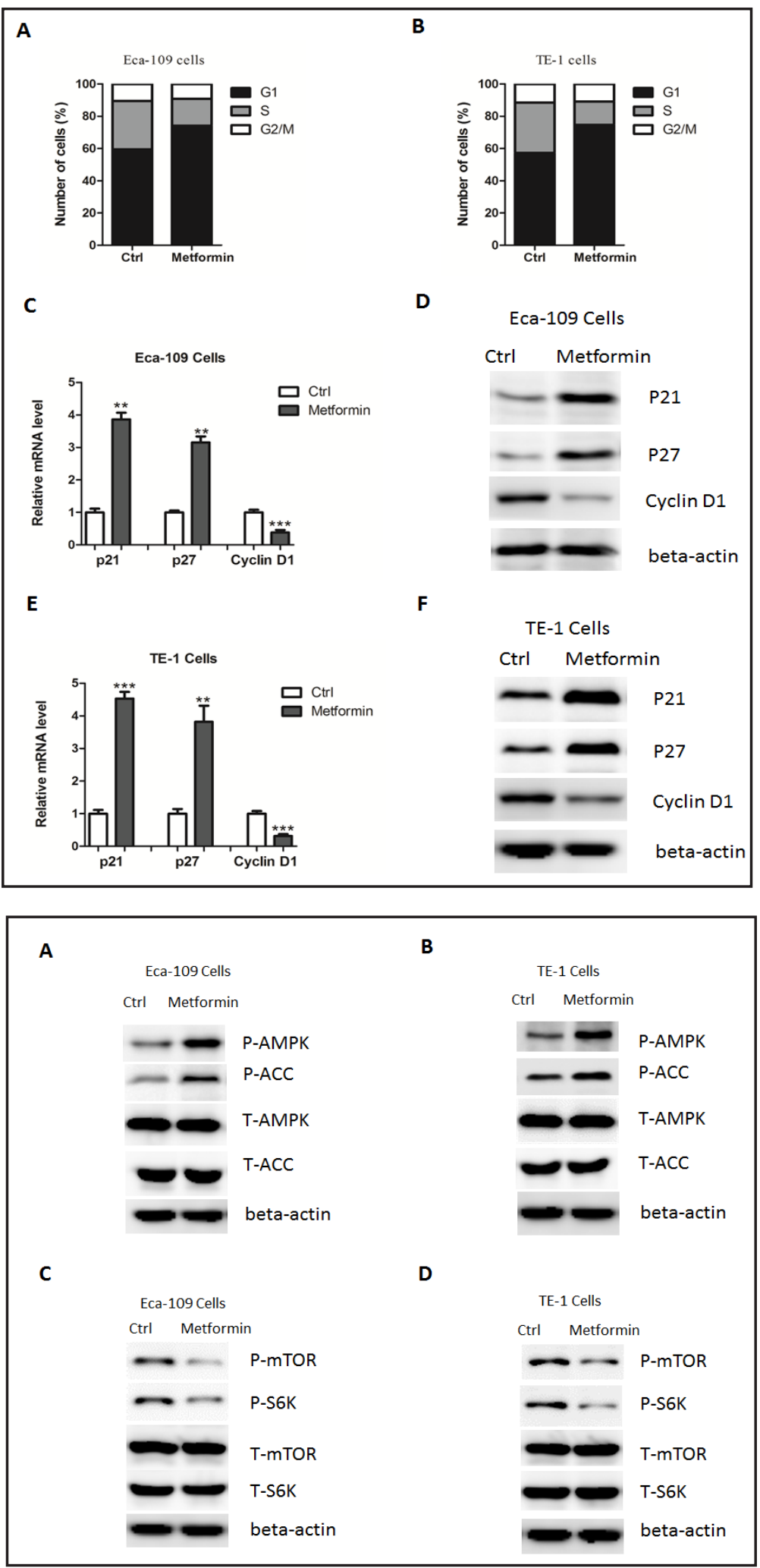

Expression of p27, p21 and Cyclin D1 protein in metformin-treated cells

We speculate that growth inhibition in esophagus cancer cells might be caused by cellcycle arrest following metformin treatment. Indeed, Eca-109 and TE-1 cells had a significantly increased percentage of cells in the G1/G0 phase and lower percentage of cells in the S phase, 
A

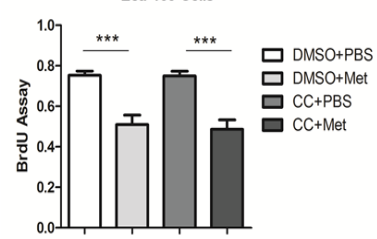

D

B
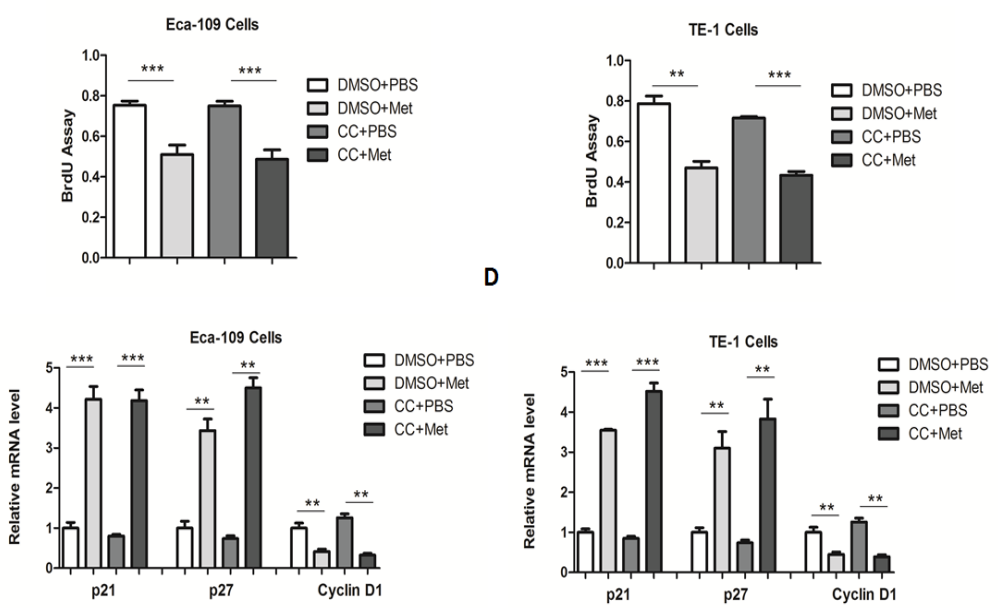

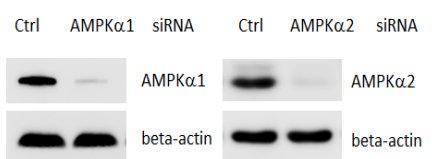

H

F

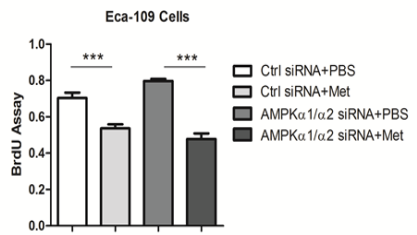

G

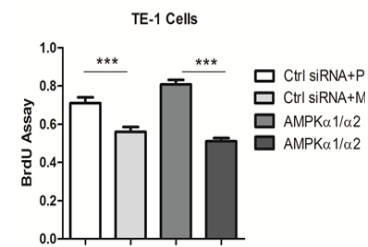

I
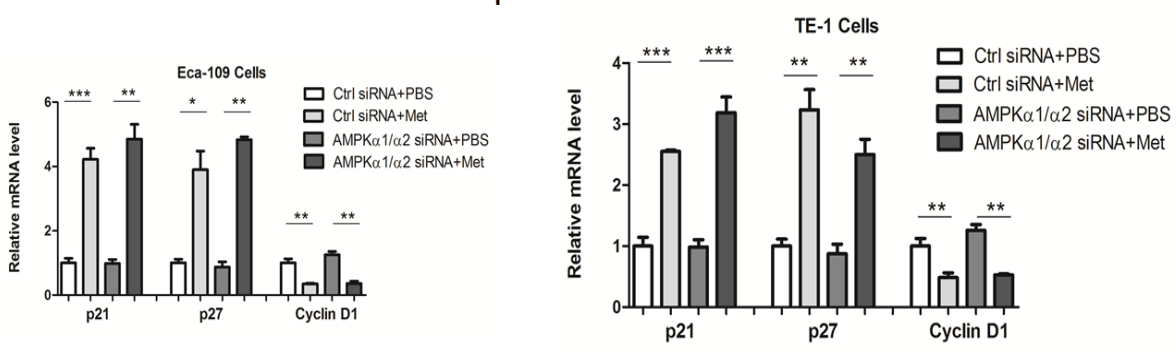

Fig. 4. The anti-proliferative action of metformin is independent of AMPK signaling activation. (A-B) Cell proliferation activity was measured by BrdU incorporation assays in Eca-109 (A) and TE-1 cells (B). Cells were pretreated with vehicle control (DMSO) or Compound C (CC) for 12 hours. (C-D) mRNA levels of p21, p27 and Cyclin D1 were determined by real-time PCR in Eca-109 (C) and TE-1 cells (D). (E) Western blot analysis of AMPK $\alpha 1$ and AMPK $\alpha 2$ in Eca-109 cells transfected with siRNA oligos against AMPK $\alpha 1 / \alpha 2$ or scramble siRNA (Ctrl). (F-G) Cell proliferation activity was measured by BrdU incorporation assays in Eca109 (F) and TE-1 cells (G). Cells were pre-transfected with siRNA oligos against AMPK $\alpha 1$ or scramble siRNA (Ctrl). (H-I) mRNA levels of p21, p27 and Cyclin D1 were determined by real-time PCR in Eca-109 (H) and TE-1 cells (I).

by $5 \mathrm{mM}$ metformin treatment (Fig. 2A-2B). Besides, we analyzed the expression contents of p21, p27 and Cyclin D1, which are known as key molecules involved in cell-cycle arrest. As shown in Fig. 2C-2F, expression levels of p21 and p27 were significantly increased in both cell lines. Besides, the contents of Cyclin D1 were markedly down-regulated in metformintreated cells (Fig. 2C-2F).

\section{Metformin up-regulates AMP kinase activity in esophagus cancer cells}

Several studies have indicated that the antiproliferative effects of metformin involved the AMP kinase pathway [11]. Indeed, our western blot analysis indicated that metformin stimulated AMPK phosphorylation in Eca-109 and TE-1cells (Fig. 3A-3B). Phosphorylated 


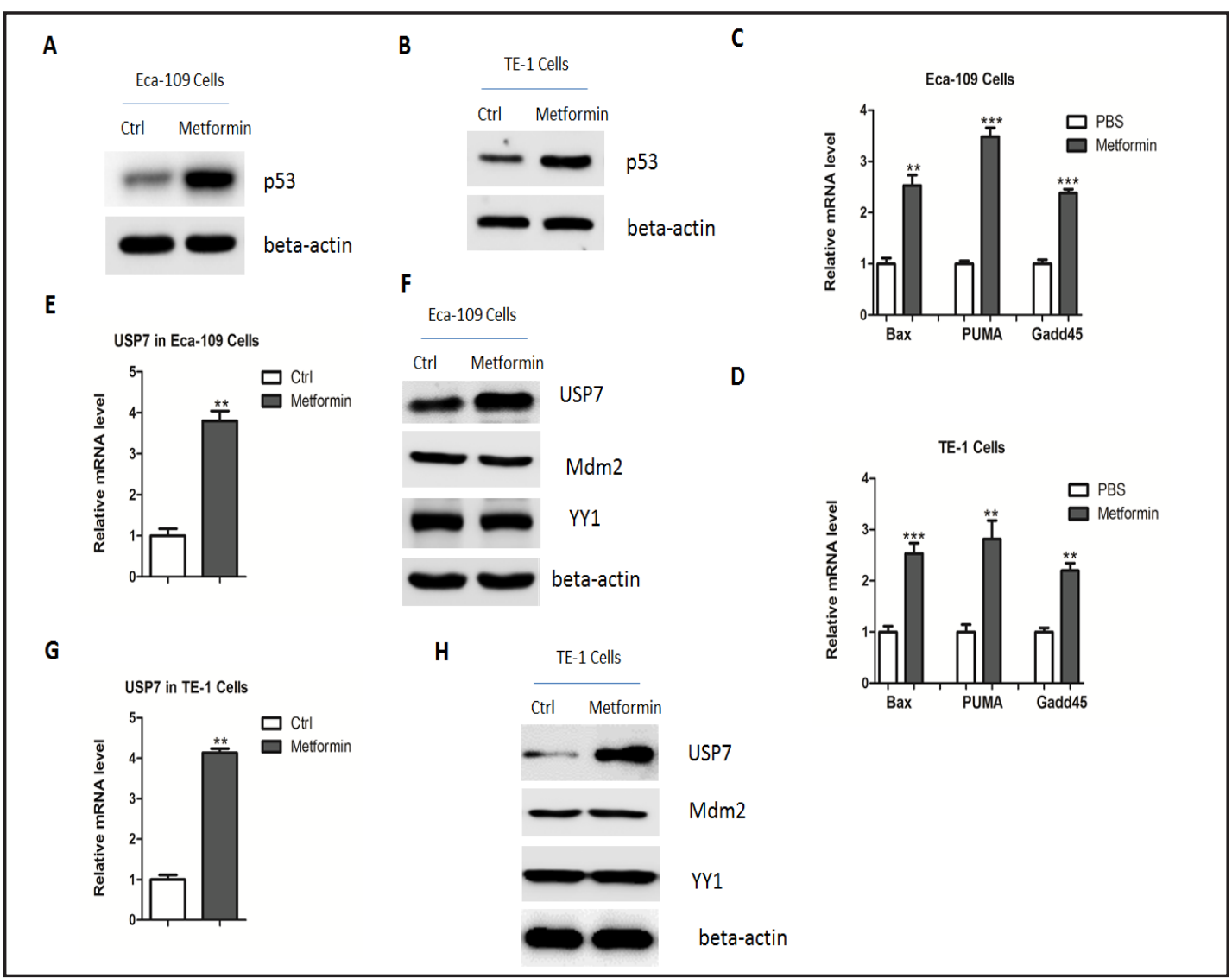

Fig. 5. Metformin increased USP7 expression in esophagus cancer cells. (A-B) Western blot analysis of USP7 expression in Eca-109 (A) and TE-1 (B) cells treated with vehicle control (PBS) or metformin. (C-D) Realtime PCR analysis of Bax, PUMA and Gadd45 expression in Eca-109 (C) and TE-1 (D) cells treated with vehicle control (Ctrl) or metformin. (E-F) mRNA (E) and protein (F) levels of USP7, YY1 and Mdm2 in Eca-109 cells treated with vehicle control (Ctrl) or metformin. (G-H) mRNA (G) and protein (H) levels of USP7, YY1 and Mdm2 in Eca-109 cells treated with vehicle control (Ctrl) or metformin.

ACC, a downstream target of AMPK, was also enhanced in cells treated with metformin (Fig. 3A-3B). Because AMPK activation inhibits energy-consuming pathways and protein synthesis, we observed that AMPK activation is associated with a decreased phosphorylation of mTOR and S6 kinase (Fig. 3C-3D).

Inhibition of AMPK pathway has little impact on the roles of metformin

We next test whether the inhibiting effect of metformin on cell proliferation is mediated by AMPK in esophagus cancer cells. As shown in Fig. 4A and 4B, pretreatment with the AMPK inhibitor (Compound C, CC) could not reverse the inhibitory effect of metformin on cell proliferation. Besides, expression levels of cell-cycle regulators were also inhibited by metformin in the presence of CC (Fig. 4C and 4D). To rule out possible nonspecific effects of CC, siRNA oligos-mediated knockdown of AMPK $\alpha 1$ and $\alpha 2$ subunit was performed (Fig. 4E). As a result, we also observed that metformin could regulate cell proliferation and expression levels of cell-cycle regulators in cells with AMPK $\alpha 1 / \alpha 2$ subunit depletion (Fig. 4F-4I). Therefore, our results suggest that the antiproliferative effects of metformin in esophagus in independent of AMPK signaling, although AMPK pathway was significantly activated by metformin treatment. 
A

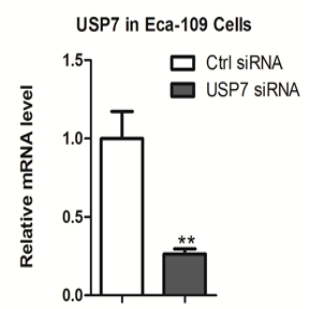

C

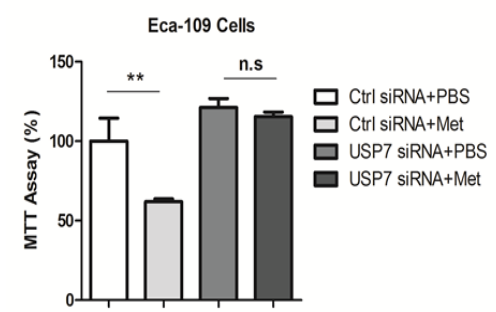

E

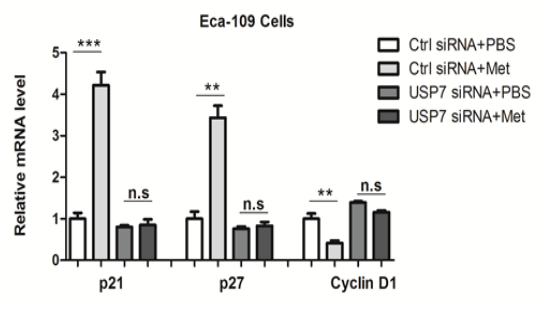

B

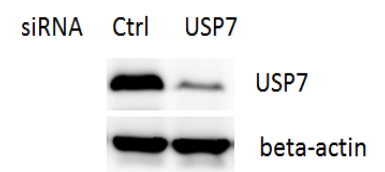

D

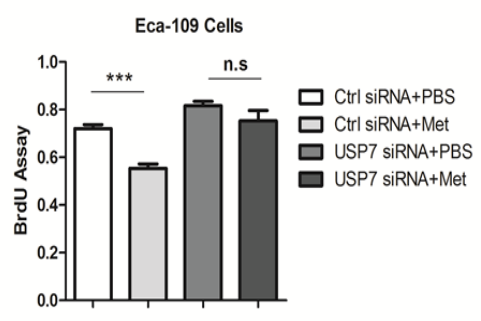

$\mathbf{F}$

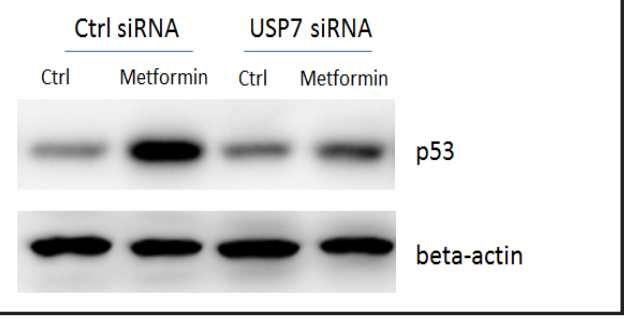

Fig. 6. USP7 deficiency reversed the anti-proliferative roles of metformin. (A-B) mRNA (A) and protein (B) levels of USP7 in Eca-109 cells transfected with siRNA oligos against USP7 or scramble siRNA (Ctrl). (C-D) Cell viability (C) and proliferation activity (D) were measured by MTT and BrdU assays in Eca-109 cells. (E) mRNA levels of p21, p27 and Cyclin D1 were determined by real-time PCR in Eca-109 cells. (F) Western blot analysis of $\mathrm{p} 53$ was determined in Eca-109 cells.

\section{Metformin increases USP7 expression and p53 protein stability}

Expression of several cell-cycle regulators including p21 and p27 is controlled by tumor suppressor p53. Given that these genes are regulated by metformin treatment in esophagus cancer cell, we analyzed p53 abundance in these cells. As shown in Fig. 5A and 5B, p53 protein contents were dramatically increased in cell treated with metformin, whereas its mRNA levels remain unchanged (data not shown). As a result, its down-stream target genes such as Bax, PUMA and Gadd45 were also up-regulated by metformin treatment (Fig. 5C and 5D).

Previous studies have demonstrated that $\mathrm{p} 53$ protein levels are regulated by several proteins such as USP7, Yin Yang 1 (YY1) and Mdm2 [21-23]. Here, we observed that USP7 expression was markedly increased after metformin treatment in Eca-109 and TE-1 cells (Fig. 5E-5H), while YY1 and Mdm2 expression remained unaffected by metformin (Fig. 5F and 5H).

SiRNA against USP7 rescues cells from metformin-induced growth inhibition.

To determine if induction of USP7 by metformin is required for the anti-proliferative effect of the drug, we carried out experiments with USP7 knockdown using siRNA oligos (Fig. 6A and 6B). As a result, the siRNA rescued cells from the inhibitory effect of metformin in Eca-109 cells (Fig. 6C and 6D). Consistently, the inhibitory functions of metformin on the expression levels of cell-cycle regulators were also reversed by USP7 siRNA oligos (Fig. 6E). 
Moreover, knockdown of USP7 reduced the protective effect of metformin on p53 protein accumulation (Fig. 6F). Similar results were also observed in TE-1 cells (data not shown). Therefore, our results highlight the USP7 as a new molecular target in anticancer therapy in response to metformin treatment.

\section{Discussion}

In the present study, we firstly explored the roles of metformin and its molecular mechanisms in esophagus cancer cells. Metformin was shown to inhibit cell prolieration in Eca-109 and TE-1 cells as evidenced by MTT and BrdU incorporation assays. Besides, metformin treatment led to a cell-cycle arrest, accompanied with up-regulation of p21 and p27 while repression of Cyclin D1 expression. Moreover, our results demonstrated that metformin activated AMP kinase activation as well as inhibition of mTOR signaling. Interestingly, inhibition of AMPK pathway using its antagonist or siRNA oligos did not affect the anti-proliferative roles of metformin. Indeed, Ben et al. revealed that antiproliferative action of metformin in prostate cancer cell lines is not mediated by AMPK, either [24]. They identified REDD1 (also known as DDIT4 and RTP801), a negative regulator of mTOR, as a new molecular target of metformin [24]. Therefore, together with their results, our data suggest that novel molecular targets in anticancer therapy in response to metformin treatment should be investigated in the future.

Here, we propose USP7 as a new molecular target of metformin in esophagus cancer cells. This is suggested by two lines of evidence. First, metformin treatment specifically upregulates USP7 mRNA and protein levels in Eca-109 and TE-1 cells. Second, USP7 invalidation, using siRNA oligos, abrogated metformin inhibition of cell proliferation. Therefore, our observations show that USP7 might be a mediator of metformin-induced cell-cycle arrest in esophagus cancer cells and is one of the components of an important pathway for tumor suppression, although the mechanisms of UPS7 up-regulation by metformin remain to be explored.

UPS7, known as the herpes simplex virus associated ubiquitin-specific protease (HAUSP), is an evolutionarily conserved protein which was originally isolated as a binding partner of the herpes simplex virus protein Vmw110/ICP0 [25]. USP7 has critical roles in the p53 tumor suppressor pathway, whereby it stabilizes p53 protein [21]. Besides, USP7 was shown to be involved in tumorigenesis [26, 27]. For instance, mRNA and protein levels of USP7 are markedly decreased in several types of cancer [28, 29]. Moreover, dys-regulation of its expression and (or) activity contributes to oncogenic transformation and is crucial for cancer development $[22,29]$. However, the roles of USP7 in esophagus cancer cells remain poorly understood, although we found that USP7 also positively regulated p53 abundance upon metformin treatment.

In conclusion, our results provide new evidence for a mechanism that may contribute to the antineoplastic effects of metformin suggested by recent population studies and justify further work to explore potential roles for it in esophagus cancer prevention and treatment.

\section{References}

1 Dawsey SP, Tonui S, Parker RK, Fitzwater JW, Dawsey SM, White RE, Abnet CC: Esophageal cancer in young people: A case series of 109 cases and review of the literature. PLoS One 2010;5:e14080.

2 Bosetti C, Levi F, Ferlay J, Garavello W, Lucchini F, Bertuccio P, Negri E, La Vecchia C: Trends in oesophageal cancer incidence and mortality in europe. Int J Cancer 2008;122:1118-1129.

3 Bailey CJ, Turner RC: Metformin. N Engl J Med 1996;334:574-579.

4 Crandall JP, Knowler WC, Kahn SE, Marrero D, Florez JC, Bray GA, Haffner SM, Hoskin M, Nathan DM: The prevention of type 2 diabetes. Nat Clin Pract Endocrinol Metab 2008;4:382-393.

5 Rotella CM, Monami M, Mannucci E: Metformin beyond diabetes: New life for an old drug. Curr Diabetes Rev 2006;2:307-315. 


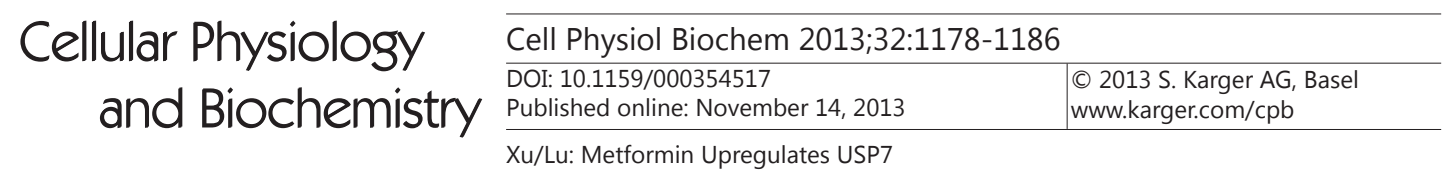

6 Hundal RS, Krssak M, Dufour S, Laurent D, Lebon V, Chandramouli V, Inzucchi SE, Schumann WC, Petersen KF, Landau BR, Shulman GI: Mechanism by which metformin reduces glucose production in type 2 diabetes. Diabetes 2000;49:2063-2069.

7 Zakikhani M, Dowling R, Fantus IG, Sonenberg N, Pollak M: Metformin is an amp kinase-dependent growth inhibitor for breast cancer cells. Cancer Res 2006;66:10269-10273.

-8 Buzzai M, Jones RG, Amaravadi RK, Lum JJ, DeBerardinis RJ, Zhao F, Viollet B, Thompson CB: Systemic treatment with the antidiabetic drug metformin selectively impairs p53-deficient tumor cell growth. Cancer Res 2007;67:6745-6752.

-9 Li D, Yeung SC, Hassan MM, Konopleva M, Abbruzzese JL: Antidiabetic therapies affect risk of pancreatic cancer. Gastroenterology 2009;137:482-488.

10 Chen HP, Shieh JJ, Chang CC, Chen TT, Lin JT, Wu MS, Lin JH, Wu CY: Metformin decreases hepatocellular carcinoma risk in a dose-dependent manner: Population-based and in vitro studies. Gut 2013;62:606-615.

11 Dowling RJ, Zakikhani M, Fantus IG, Pollak M, Sonenberg N: Metformin inhibits mammalian target of rapamycin-dependent translation initiation in breast cancer cells. Cancer Res 2007;67:10804-10812.

12 Kisfalvi K, Eibl G, Sinnett-Smith J, Rozengurt E: Metformin disrupts crosstalk between g protein-coupled receptor and insulin receptor signaling systems and inhibits pancreatic cancer growth. Cancer Res 2009;69:6539-6545.

13 Hirsch HA, Iliopoulos D, Struhl K: Metformin inhibits the inflammatory response associated with cellular transformation and cancer stem cell growth. Proc Natl Acad Sci U S A 2013;110:972-977.

14 Anisimov VN, Egormin PA, Piskunova TS, Popovich IG, Tyndyk ML, Yurova MN, Zabezhinski MA, Anikin IV, Karkach AS, Romanyukha AA: Metformin extends life span of HER-2/neu transgenic mice and in combination with melatonin inhibits growth of transplantable tumors in vivo. Cell Cycle 2010;9:188-197.

15 Zhou G, Myers R, Li Y, Chen Y, Shen X, Fenyk-Melody J, Wu M, Ventre J, Doebber T, Fujii N, Musi N, Hirshman MF, Goodyear LJ, Moller DE: Role of AMP-activated protein kinase in mechanism of metformin action. J Clin Invest 2001;108:1167-1174.

16 Musi N, Hirshman MF, Nygren J, Svanfeldt M, Bavenholm P, Rooyackers O, Zhou G, Williamson JM, Ljunqvist O, Efendic S, Moller DE, Thorell A, Goodyear LJ: Metformin increases AMP-activated protein kinase activity in skeletal muscle of subjects with type 2 diabetes. Diabetes 2002;51:2074-2081.

17 Green AS, Chapuis N, Lacombe C, Mayeux P, Bouscary D, Tamburini J: LKB1/AMPK/mTOR signaling pathway in hematological malignancies: From metabolism to cancer cell biology. Cell Cycle 2011;10:21152120.

18 Rattan R, Graham RP, Maguire JL, Giri S, Shridhar V: Metformin suppresses ovarian cancer growth and metastasis with enhancement of cisplatin cytotoxicity in vivo. Neoplasia 2011;13:483-491.

19 Foretz M, Hebrard S, Leclerc J, Zarrinpashneh E, Soty M, Mithieux G, Sakamoto K, Andreelli F, Viollet B: Metformin inhibits hepatic gluconeogenesis in mice independently of the LKB1/AMPK pathway via a decrease in hepatic energy state. J Clin Invest 2010;120:2355-2369.

20 Kalender A, Selvaraj A, Kim SY, Gulati P, Brule S, Viollet B, Kemp BE, Bardeesy N, Dennis P, Schlager JJ, Marette A, Kozma SC, Thomas G: Metformin, independent of AMPK, inhibits mTORC1 in a rag GTPasedependent manner. Cell Metab 2010;11:390-401.

21 Sarkari F, Sheng Y, Frappier L: USP7/HAUSP promotes the sequence-specific DNA binding activity of p53. PLoS One 2010;5:e13040.

-22 Epping MT, Meijer LA, Krijgsman O, Bos JL, Pandolfi PP, Bernards R: TSPYL5 suppresses p53 levels and function by physical interaction with USP7. Nat Cell Biol 2011;13:102-108.

23 Sui G, Affar el B, Shi Y, Brignone C, Wall NR, Yin P, Donohoe M, Luke MP, Calvo D, Grossman SR, Shi Y: Yin Yang 1 is a negative regulator of p53. Cell 2004;117:859-872.

24 Ben Sahra I, Regazzetti C, Robert G, Laurent K, Le Marchand-Brustel Y, Auberger P, Tanti JF, GiorgettiPeraldi S, Bost F: Metformin, independent of AMPK, induces mTOR inhibition and cell-cycle arrest through REDD1. Cancer Res 2011;71:4366-4372.

25 Everett RD, Meredith M, Orr A, Cross A, Kathoria M, Parkinson J: A novel ubiquitin-specific protease is dynamically associated with the pml nuclear domain and binds to a herpesvirus regulatory protein. EMBO J 1997; 16:1519-1530.

-26 Shi D, Grossman SR: Ubiquitin becomes ubiquitous in cancer: emerging roles of ubiquitin ligases and deubiquitinases in tumorigenesis and as therapeutic targets. Cancer Biol Ther 2010;10:737-747.

27 Maertens GN, El Messaoudi-Aubert S, Elderkin S, Hiom K, Peters G: Ubiquitin-specific proteases 7 and 11 modulate Polycomb regulation of the INK4a tumour suppressor. EMBO J 2010;29:2553-2565.

28 Yang HW, Kim TM, Song SS, Shrinath N, Park R, Kalamarides M, Park PJ, Black PM, Carroll RS, Johnson MD: Alternative splicing of CHEK2 and codeletion with NF2 promote chromosomal instability in meningioma. Neoplasia 2012;14:20-28.

29 Yang Z, Huo S, Shan Y, Liu H, Xu Y, Yao K, Li X, Zhang X: STAT3 repressed USP7 expression is crucial for colon cancer development. FEBS Lett 2012;586:3013-3017. 